\title{
Structural Characterization and Photoelectrochemical Properties of Silver Nanoparticle-Polyion Films
}

\author{
Taichi Arakawa ${ }^{1}$, Tsuyoshi Akiyama ${ }^{1,2^{*}}$ and Sunao Yamada ${ }^{1,2,3^{*}}$ \\ ${ }^{\text {I}}$ Department of Materials Physics and Chemistry, Graduate School of Engineering, Kyushu University, Moto-oka 744, \\ Nishi-ku, Fukuoka 819-0395, Japan \\ Fax: +81-92-802-2815, e-mail: taichi13tcm@mbox.nc.kyushu-u.ac.jp \\ ${ }^{2}$ Department of Applied Chemistry, Graduate School of Engineering, Kyushu University, Moto-oka 744, Nishi-ku, \\ Fukuoka 819-0395, Japan
}

Fax: +81-92-802-2815, e-mail: t-akitcm@mbox.nc.kyushu-u.ac.jp

${ }^{3}$ Center for Future Chemistry, Kyushu University, Moto-oka 744, Nishi-ku, Fukuoka 819-0395, Japan

Fax:+81-92-802-2815, e-mail: sunaotem@mbox.nc.kyushu-u.ac.jp

Fabrication of silver nanoparticle (AgP) - polyion alternate films were successful by using layer-by-layer technique. Alternate films of AgPs and polyion were fabricated as follows. First, a quartz glass substrate or an indium-tin-oxide (ITO) substrate was poured into the solution of polyethyleneimine (PEI) and then polystylenesulfonate (PSS). Then, the PSS/PEI- modified substrate was dipped into the solution of PEI and AgPs to give alternate assemblies of AgP-PEI on the PEI/PSS substrate. Adsorption of AgPs was characterized by transmission absorption spectroscopy and quartz crystal microbalance measurements. Morphologies of films were investigated by scanning electron microscopy and X-ray photoelectron spectroscopy. These results indicate the achievement of stepwise adsorption of AgPs and the formation of layer-by-layer structure. It was also observed that the films fabricated on the ITO electrode showed appreciable photocurrent based on photoexitation of AgPs.

Key words: layer-by-layer, silver nanoparticle, alternate assembly, plasmon band, photocurrent

\section{INTRODUCTION}

During few decades, metal nanoparticles have attracted much attention due to their unique optical properties that are different from those in bulk metal. Especially, gold nanoparticles and silver nanoparticles ( $\mathrm{AgPs}$ ) have optical properties caused by the interaction with light in the ultraviolet to near infrared region and show intense absorption and generate strong electromagnetic field ${ }^{1)}$, and thus they have been expected for applications such as coloring 2,3 , surface-enhanced Raman scattering $(\mathrm{SERS})^{4,5)}$, and metal-enhanced fluorescence (MEF). ${ }^{6,7)}$ Particularly, AgPs show large enhancement factors of electromagnetic field ${ }^{8)}$, high conductivity ${ }^{9)}$, antibacterial characteristic ${ }^{10)}$, and so on.

There are many methods to fabricate thin solid films, for example, surface sol-gel ${ }^{11,12)}$, electrochemical polymerization ${ }^{13,14)}$, and layer-by-layer (LBL) techniques ${ }^{15 \sim 22)}$. Among them, the LBL technique is convenient and easy to control the thickness of films as well as the amount of metal nanoparticles. For example, Goulet and Aroca fabricated multilayered films of citrate-protected AgPs and a dendrimer. ${ }^{16)}$

Meanwhile, investigation of photoelectric conversion using AgPs has been carried out. Since AgPs have higher conductivity and larger absorption coefficient than the other metal nanoparticle, AgPs will be expected to function as the nanostructured electrode as well photoabsorbing materials. Recently, $\mathrm{He}$ and Yamagishi reported the unique photocurrent responses from $\mathrm{TiO}_{2}$-pigment-AgP multilayered films. ${ }^{22}$ Tian and Tatsuma observed anodic photocurrent generation in $\mathrm{TiO}_{2}$-AgPs films derived from plasmon absorption of AgPs. ${ }^{23)}$ The LBL technique, on the other hand, may be superior for rapid fabrication and control of the degree of stacked AgP assemblies.

In this paper, we have fabricated multilayered films of AgPs capped with citrate, by using the layer-by-layer technique and demonstrated photocurrent generation from the film.

\section{EXPERIMENTAL PROCEDURE}

Silver nitrate $\left(\mathrm{AgNO}_{3}\right)$, poly(styrenesulfonate) (PSS, 
$\mathrm{Mw}=70000)$, poly(ethyleneimine) $(\mathrm{PEI}, \mathrm{Mw}=$ 50000-100000), and other chemicals were used as received. AgPs capped with citrate were synthesized according to the modified procedure of Lee. ${ }^{24)}$ The mean diameter of AgPs was determined to be $55 \pm 12 \mathrm{~nm}$, using a JEOL JEM-200CX transmission electron microscope (TEM). Briefly, $90 \mathrm{mg}$ of $\mathrm{AgNO}_{3}$ was dissolved into 500 $\mathrm{ml}$ of $\mathrm{H}_{2} \mathrm{O}$. After refluxing, $10 \mathrm{ml}$ of sodium citrate solution (10wt\%) was added and then refluxed for 30 min.

A quartz glass substrate was immersed in conc. $\mathrm{H}_{2} \mathrm{SO}_{4}$ (97\%) for a few days and then sonicated in a mixed solution of ethanol (EtOH) and $1 \mathrm{wt} \%$ aqueous $\mathrm{KOH}$ solution $(40 \%)$. Finally, the substrate was washed with $\mathrm{H}_{2} \mathrm{O}$ and dried in nitrogen gas flow. The ITO electrode was washed with acetone and ethanol, then cleaned by ozone etching for $15 \mathrm{~min}$, followed by washing with $\mathrm{H}_{2} \mathrm{O}$ and then drying with the nitrogen gas.

The preparation procedures of $\mathrm{AgP}$ films are shown in Fig. 1. First, the quartz glass substrate was immersed into $1.5 \mathrm{mM}$ PEI aqueous solution for $20 \mathrm{~min}$ at $30^{\circ} \mathrm{C}$ (step 1). Then, it was immersed into $3 \mathrm{mM}$ PSS aqueous solution for $20 \mathrm{~min}$ at $30^{\circ} \mathrm{C}$ (step 2), to give the glass plate modified with PEI and PSS as: PSS/PEI/Glass. After withdrawal of each step, the substrate was washed with $\mathrm{H}_{2} \mathrm{O}$ and dried with the nitrogen gas. Next, PSS/PEI/glass was immersed into the PEI solution (step 3), followed by washing with $\mathrm{H}_{2} \mathrm{O}$ and drying with the nitrogen gas, giving polyion-modified glass substrate: (PEI/PSS/PEI/Glass). Then, PEI/PSS/PEI/Glass was immersed into the aqueous colloidal solution of AgPs for $12 \mathrm{~h}$ at $30^{\circ} \mathrm{C}$ (step 4). AgPs, capped with citrate, were electrostatically adsorbed on the outermost cationic layer, giving a AgP-deposited sample as: AgP/PEI/PSS/PEI/Glass. By repeating steps 3 and 4 , multilayered films of AgPs and PEI, $(\mathrm{AgP} / \mathrm{PEI})_{\mathrm{n}} / \mathrm{PSS} / \mathrm{PEI} / \mathrm{Glass}(\mathrm{n}=1-5)$, were fabricated.

Transmission absorption spectra of

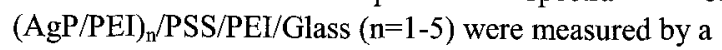
JASCO V-670 UV-vis near IR (NIR) spectrophotometer using the integrating sphere. Adsorbed amounts of PEI and AgPs were measured by the quartz crystal microbalance (QCM) technique using an ALS MODEL 420 electrochemical QCM. The QCM tip (AT-cut, 8 $\mathrm{MHz}$ ) was modified with 2-hydroxyethanethiol before fabricating films. Scanning electron microscope (SEM) observation was carried out with HITACHI S-5000. The $\mathrm{X}$-ray photoelectron spectroscopy (XPS) was done by a PHI-5800 spectrometer. Photocurrent measurements were carried out in an aqueous solution containing $0.1 \mathrm{M}$ $\mathrm{NaClO}_{4}$, using three-electrode photoelectrochemical cell with $\mathrm{Ag} / \mathrm{AgCl}$ (sat. $\mathrm{KCl}$ ) as a reference electrode and a platinum wire as a counter electrode, respectively. As a working electrode, PEI/PSS/PEI/ITO, or $(\mathrm{AgP} / \mathrm{PEI})_{\mathrm{n}} / \mathrm{PSS} / \mathrm{PEI} / \mathrm{ITO} \quad(\mathrm{n}=1,3, \quad$ or 5$)$ was independently fabricated according to the procedures of Fig. 1. In all measurements, triethanolamine (TEOA) $\left(5.0 \times 10^{-2} \mathrm{M}\right)$ was added to the electrolyte solution as a sacrificial reagent. The monochromatic light from a Xe lamp irradiated the modified electrode, and the steady-state photocurrents were measured using a Huso HECS-318C potentiostat.

\section{RESULTS AND DISCUSSION}

Transmission absorption spectra of (AgP/PEI) $/$ /PSS/PEI/Glass ( $\mathrm{n}=1-5)$ are shown in Fig. 2. In each spectrum, a broad absorption peak at $\sim 380 \mathrm{~nm}$, based on the plasmon oscillation of isolated AgPs or transverse plasmon mode of coupled particles, and a broad band around $700 \mathrm{~nm}$ deriving from the plasmon oscillation of interparticle plasmon coupling were observed. ${ }^{16)}$ Both bands increased with increasing the number of set of AgP-PEI deposition (steps 3 and 4). A sharp absorption dip at $\sim 380 \mathrm{~nm}$ was always observed in the sample. It tended to increase with increasing the number of a set of PEI-AgP deposition, though the reason is not clear at this stage. Thus, it was confirmed that not only two dimensional interactions, but also three dimensional interactions among AgPs in the layers were verified.
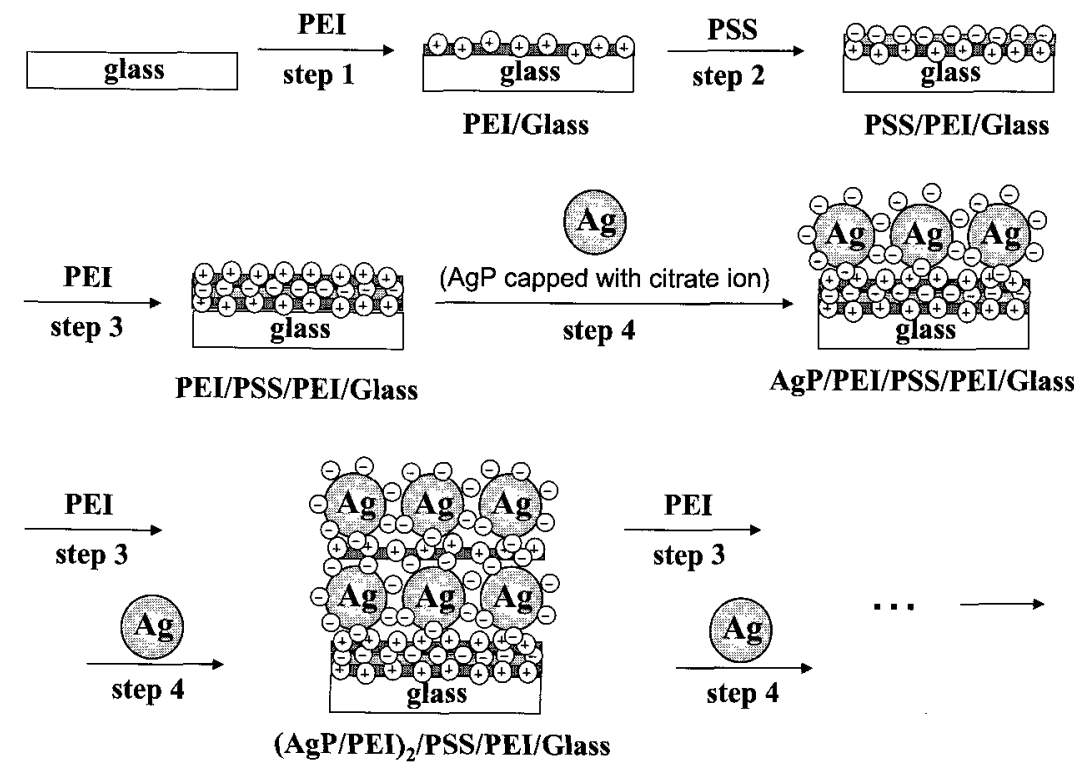

Fig. 1. Preparation procedures of alternate assemblies 


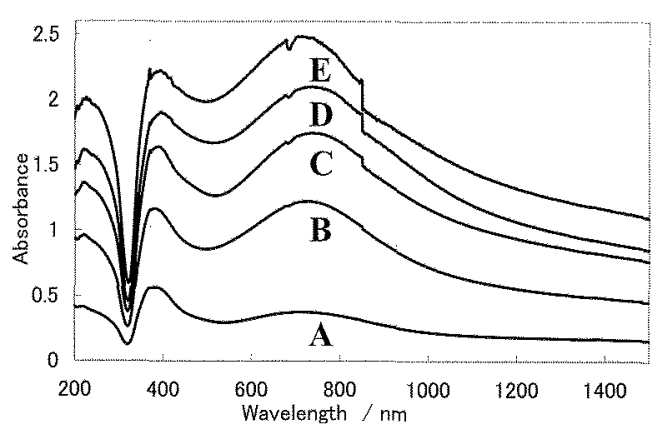

Fig. 2. Transmission absorption spectra of alternate films, $(\mathrm{AgP} / \mathrm{PEI})_{\mathrm{n}} \mathrm{PSS} / \mathrm{PEI} /$ Glass:

$A, n=1 ; B, n=2 ; C, n=3 ; D, n=4 ; E, n=5$

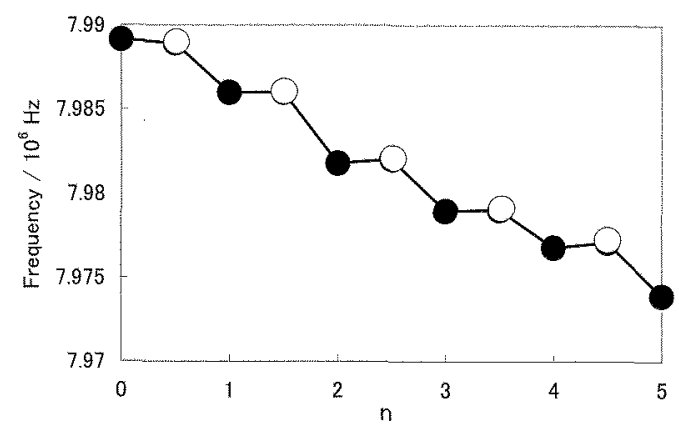

Fig. 3. QCM frequency changes after alternate immersing into the aqueous PEI solution ( $\bigcirc$ ) and the AgP colloidal solution ( $)$. The abscissa " $n$ " indicates the number of a set of of PEI-AgP assembling.

The results of QCM measurement are shown in Fig. 3. The stepwise frequency decrease (mass increase) after immersing into the colloidal solution of AgPs clearly indicates the adsorption of $\mathrm{AgPs}$ on the substrate. From the analysis of frequency decrease, the mean coverage of AgPs is evaluated to the $1.6 \times 10^{10}$ particles $/ \mathrm{cm}^{2}$, corresponding to about $40 \%$ of fractional coverage assuming hexagonal packing. On the other hand, slight frequency decrease (mass increase) was observed after immersing into the PEI aqueous solution. This probably indicates the trivial desorption of deposited AgPs on immersion into the PEI aqueous solution.

SEM images of $(\mathrm{AgP} / \mathrm{PEI})_{\mathrm{n}} / \mathrm{PSS} / \mathrm{PEI} / \mathrm{Glass}(\mathrm{n}=1,5)$ are shown in Fig. 4. There are many bright spots of AgPs for $\mathrm{AgP} / \mathrm{PEI} / \mathrm{PSS} / \mathrm{PEI} / \mathrm{Glass}$, which confirm the adsorption of AgPs on the substrate. On the other hand,

(a)

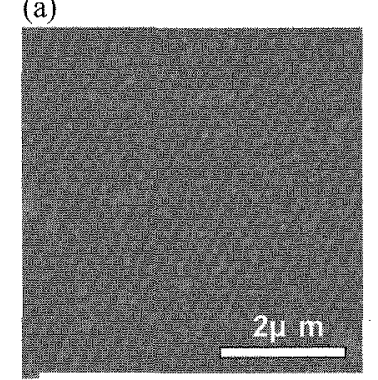

(b)

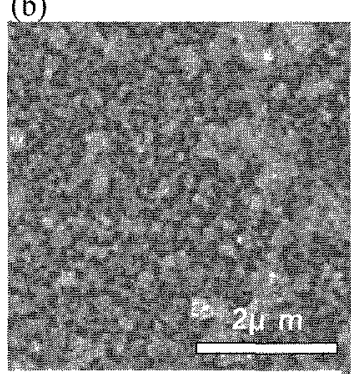

Fig. 4. SEM images of alternate films,

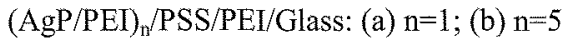

considerable aggregation of AgPs is observed for $(\mathrm{AgP} / \mathrm{PEI})_{5} / \mathrm{PSS} / \mathrm{PEI} / \mathrm{Glass}$. These results are quite consistent with the results of transmission absorption spectra (Fig. 2).

To clarify the structure of the assemblies, the atomic concentrations of $(\mathrm{AgP} / \mathrm{PEI})_{\mathrm{n}} / \mathrm{PSS} / \mathrm{PEI} / \mathrm{Glass}(\mathrm{n}=3,4)$, $\mathrm{PEI} /(\mathrm{AgP} / \mathrm{PEI})_{3} / \mathrm{PSS} / \mathrm{PEI} / \mathrm{Glass}$ were measured by XPS spectroscopy. As is summarized in Table 1, the photoelectron intensity of N1s mainly derived from PEI increased from 9.76 to 12.98 (relative intensity), and $\mathrm{Ag} 3 \mathrm{~d}$ derived from AgPs decreased from 15.35 to 12.47 after immersion into the PEI aqueous solution. Subsequent decrease of N1s from 12.98 to 7.06 and increase of $\mathrm{Ag} 3 \mathrm{~d}$ from 12.47 to 23.32 were observed after immersion into the colloidal solution of AgPs. These results clearly indicate that the LBL structure of PEI and AgPs is formed by repeating the alternate immersion processes.

Table I. Atomic concentrations of alternate films

\begin{tabular}{ccccc}
\hline & Cls & N1s & O1s & Ag3d \\
\hline (PEI/AgP $)_{3} /$ PSS/PEI/glass & 55.10 & 9.76 & 19.79 & 15.35 \\
PEI/(PEI/AgP $)_{3} /$ PSS $/$ PEI /glass & 55.44 & 12.98 & 19.11 & 12.47 \\
$(\text { PEI/AgP })_{4} /$ PSS/PEI/glass & 50.09 & 7.06 & 19.53 & 23.32 \\
\hline
\end{tabular}

Fig. 5 shows photocurrent action spectra of ITO, $\mathrm{PEI} / \mathrm{PSS} / \mathrm{PEI} / \mathrm{ITO}$, and $(\mathrm{AgP} / \mathrm{PEI})_{\mathrm{n}} / \mathrm{PSS} / \mathrm{PEI} / \mathrm{ITO}(\mathrm{n}=1,3$, 5) at $\mathrm{OV}$ versus $\mathrm{Ag} / \mathrm{AgCl}$ (sat. $\mathrm{KCl}$ ), respectively. The photocurrent increased in the anodic direction, as a set of PEI-AgP deposition proceeded $(\boldsymbol{\Delta}, \boldsymbol{\bullet}, \mathbf{D})$, as shown in the inset. These results indicate that incorporation of AgPs into the films certainly enhances the photocurrent in the anodic direction. The ITO itself showed anodic photocurrent $(\triangle)$, but its intensity was two orders of magnitude lower than the AgP-modified samples, and thus it is negligible in the present case. Also, it is not clear at this stage for slight generation of photocurrent from PEI/PSS/PEI/ITO, in spite of absence of AgPs (O). However, the photocurrent was still extremely low as compared with the AgP-modified samples.

There seems to be two photoexcitation processes for photocurrent generation: photoexcitation of ITO electrode and AgPs. As to AgP-ITO system, reverse electron-transfer from ITO (electrode) to the electron-lacked $\mathrm{AgP}$ occurs competitively with the corresponding forward electron-transfer. ${ }^{25)}$ This leads to the reduction of photocurrent generation from the AgP-ITO system when the plasmon band of AgP is excited. Meanwhile, photoexcitation of inner-core electrons of $\mathrm{AgP}$, where work functions are larger than the energy of surface plasmon oscillation, may enable electron-injection from photoexcited $\mathrm{AgP}$ to the conduction band of ITO $(>\sim 3.1 \mathrm{eV})$. Accordingly, it is suggested that the photocurrent profiles observed in the present films are based on the photoexcitation of the inner-core electrons rather than that of the free electrons. In order to verify the mechanism, we are changing the electrode materials as well as other metal nanoparticles. 


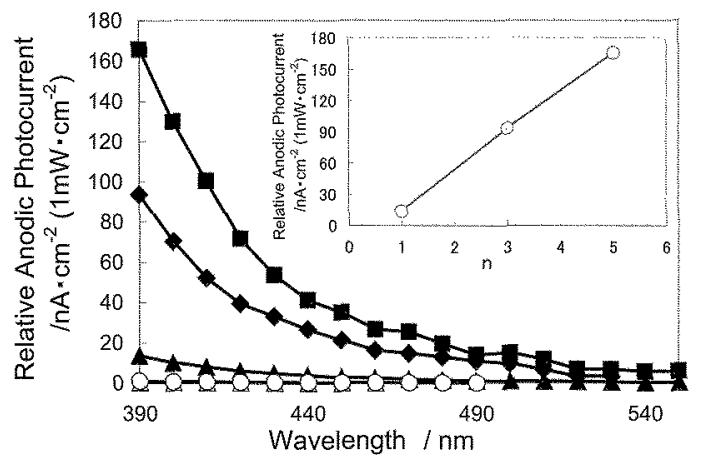

Fig. 5. Photocurrent action spectra of ( $\triangle$ ) ITO, (O) PEI/PSS/PEI/ITO, (4) AgP/PEI/PSS/PEJ/Glass, (ヤ) $(\mathrm{AgP} / \mathrm{PEI})_{3} / \mathrm{PSS} / \mathrm{PEI} /$ Glass, (a) $(\mathrm{AgP} / \mathrm{PEI})_{5} / \mathrm{PSS} / \mathrm{PEI} / \mathrm{Glass}$ (Inset; Photocurrent at 390nm. The abscissa " $\mathrm{n}$ " indicates the number of a set of of PEI-AgP assembling.)

\section{CONCLUSIONS}

We have succeeded in the alternate assembling of citrate-capped AgPs and polyions on a quartz or an ITO glass by using the LBL technique. Stepwise adsorption of AgPs onto the PEI-modified substrate was confirmed by transmission absorption, QCM, SEM observation. The AgP-modified sample clearly showed anodic photocurrent generation, induced by photoexcitation of the plasmon oscillation of deposited AgPs. Since the LBL technique is rapid for relatively homogeneous particle deposition, and needs no sophisticated instruments, the combination of some different metal nanoparticles is quite interesting. The work is in progress along this line.

Acknowledgement

This study was partially supported by Grant-in-Aid for Young Scientists (A) (18685023) and on Priority Area (Area code 470, Strong Photons-Molecules Coupling Fields : No. 19049012) from the Ministry of Education, Culture, Sports, Science and Technology of Japan.

[1] G. Mie, Ann. Phys., 25, 377-455 (1908)

[2] Y. Ohko, T. Tatsuma, T. Fujii, K. Naoi, C. Niwa, Y. Kubota, A. Fujishima, Nat. Mater., 2, 29-31 (2003)

[3] J. Liu, Y. Lu, J. Am. Chem. Soc., 127, 12677-83 (2005)

[4] K. Kneipp, Y. Wang, H. Kneipp, L. T. Perelman, I. Itzkan, R. R. Dasari, M. S. Feld, Phys. Rev. Lett., 78, 1667-70 (1997)

[5] S. Nie, S. Emory, Science, 275, 1102-06 (1997)

[6] T. Akiyama, M. Nakada, N. Terasaki, S. Yamada, Chem. Commun., 395-397 (2006)

[7] T. Akiyama, T. Kawahara, T. Arakawa, S. Yamada, submitted.

[8] A. O. Govorov, I. Carmeli, Nano. Lett., 7, 620-5 (2007)

[9] B. Houng, Appl. Phys. Lett., 87, 251922 (2005)
[10] P. Podsiadlo, S. Patemel, Jean-Marie Rouillard, Z. Zhang, J. Lee, Jung-Woo Lee, E. Gulari, N. A. Kotov, Langmuir, 21, 11915-21 (2005)

[11] T. Akiyama, K. Matsuoka, T. Arakawa, K. Kakutani, A. Miyazaki, S. Yamada, Jpn. J. Appl. Phys., 45(4B), 3758-61 (2006)

[12] T. Arakawa, T. Kawahara, T. Akiyama, S. Yamada, Jpn. J. Appl. Phys., 46(4B), 2490-92 (2007)

[13] K. Sugawa, K. Kakutani, T. Akiyama, S. Yamada, K. Takechi, T. Shiga, T. Motohiro, K. Kohama, Jpn. J. Appl. Phys., 46(4B), 2632 (2007)

[14] K. Sugawa, T. Akiyama, S. Yamada, Thin Solid Films, in press.

[15] J. Schmitt, G. Decher, Adv. Mater., 9, 61-5 (1997)

[16] P. J. G. Goulet, D. S. dos Santos, Jr., R. A. Alvarez-Puebla, O. N. Oliveira, Jr., R.F. Aroca, Langmuir, 21, 5576-81 (2005)

[17] N. P. W. Pieczonka, P. J. G. Goulet, R. F. Aroca, J. Am. Chem. Soc., 128, 12626-7 (2006)

[18] X. Li, W. Xu, J. Zhang, H. Jia, B. Yang, B. Zhao, B. Li, Y. Ozaki, Langmuir, 20, 1298-304 (2004)

[19] X. Zou, H. Bao, H. Guo, L. Zhang, L. Qi, J. Jiang, L.Niu, S. Dong, J. Colloid Interface Sci., 295, 401-8 (2006)

[20] T. Cassagneau, J. H. Fendler, J. Phys. Chem. B, 103, 1789-93 (1999)

[21] S. Zhao, K. Zhang, J. An, Y. Sun, C. Sun, Mater. Lett., 60, 1215-8 (2006)

[22] J. He, P. Yang, H. Sato, Y. Umemura, A. Yamagishi, J. Electroanal. Chem., 566, 227-33 (2004)

[23] Y. Tian, T. Tatsuma, Chem. Commun., 1810-1 (2004)

[24] P. C. Lee, D. Meisel, J. Phys. Chem., 86, 3391-5 (1982)

[25] K. Kawahara, K.Suzuki, Y. Ohko, T. Tatsuma, Phys. Chem. Chem. Phys., 7, 3851 (2005)

(Received December 8, 2007;Accepted Febrary 12, 2008) 